\title{
Context-Aware Services for Physical Hypermedia Applications
}

\author{
Gustavo Rossi $^{1,2, *}$, Silvia Gordillo ${ }^{1,3}$, Cecilia Challiol $^{1}$, and Andrés Fortier ${ }^{1}$ \\ ${ }^{1}$ LIFIA. Facultad de Informática. UNLP. La Plata, Argentina \\ \{gustavo, gordillo, ceciliac, andres\}@lifia.info.unlp.edu.ar
${ }^{2}$ Also CONICET, ${ }^{3}$ Also CICPBA
}

\begin{abstract}
In this paper we present an approach for designing and deploying context-aware services in the context of physical hypermedia applications, those applications in which mobile users explore real and digital objects using the hypermedia paradigm. We show how to adapt the objects' response to the user's navigation context by changing the role these objects play in the user's travel. We first motivate our research with a simple example and survey some related work; next we introduce the concept of travel object and show that physical objects might assume the role of different type of travel objects. We then present an architectural approach for context-aware services and describe its evolution into a software substrate for physical hypermedia services. We conclude by indicating some further work we are pursuing.
\end{abstract}

\section{Introduction and Background}

Physical Hypermedia (PH) applications extend the well-known navigation paradigm, made popular by the Web, in order to support the mobile user and to allow a unified exploration of digital and physical (real-world) objects. In a typical PH application physical objects are augmented with digital information in such a way that when the user is sensed to be facing an object it can access information on this object (as if it were a hypermedia node) in his mobile device. From this point of view, one can consider PH application as a particular case of Location-aware software; however physical objects may also exhibit hypermedia links to other objects (either physical or digital). The user explores these links and might either get digital information (when the link's target object is digital) or some indication on how to "walk" the link to reach the physical target. More elaborated context-aware responses can be provided by personalizing the information (or links) made available to the user, to his actual context, preferences, etc.

This simple but powerful idea can be very appealing in many different domains, such as tourism, entertainment, logistics, etc. In fact every former application of the "old" hypermedia paradigm, in which the user is mobile can benefit from these concepts. Many authors have already shown the feasibility of this kind of software (certainly an evolution of mobile information systems) in different areas such as learning

\footnotetext{
* This work has been partially funded by Project PICT 2003, Nro 13623, SeCyT. 
games [16], design [8], tourism [10], collaboration [3], and have built powerful frameworks to support the construction of hybrid information networks in which the real and the digital worlds are glued together with links.

Imagine for example a tourist application for a city (we exemplify here with our city, La Plata). When the user is in front of the Cathedral he accesses the information in the hypermedia node corresponding to this physical object. He can explore links which allow him to read about the building process, the historical context in which it was created, etc. Some links "point" to other buildings by the same architect. These links are called in our modeling approach "walkable" links (WLinks) [6]; when the user selects one of them, he is presented a plan to reach the building. In his tour, he can either reach the target or deviate because he found another interesting place to explore; when facing other physical objects, they can help him to find his way, show him information, etc. An oversimplified schema of this example can be seen in Figure 1. The thick black line indicates the relationship which the user is exploring by walking from the Cathedral to the Town Hall.

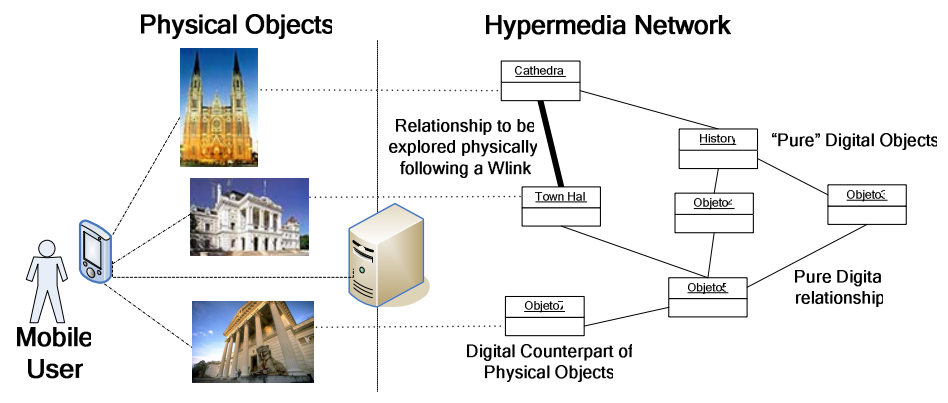

Fig. 1. A Schematic View of Physical Hypermedia

Our research in this area has included the definition of a modeling approach which extends the OOHDM hypermedia design framework [14] to this field [7] and an implementation framework which extends a standard Web application architecture to support physical objects and mobile users [1]. In this paper we present a seamless extension to an architecture for context-aware services [7] to make it PH "compliant", i.e. to support navigation and hypermedia-aware context information; we also discuss the concept of context-aware hypermedia services and show how we materialized these services in our architecture.

The main contributions of our paper are the following:

- We present the concept of context-aware PH services and relate it with the role which physical objects play in a user's journey.

- We present a modular implementation of these ideas stressing the need to keep our base architecture evolvable to support further extensions in this field.

The rest of the paper is organized as follows: In Section 2 we briefly discuss related work. In Section 3, we discuss the idea of physical hypermedia services and present a model of travel objects; In Section 4 we give an overview of our architecture 
for context-aware services; we focuses on our solution for building physical hypermedia services and show some simple but meaningful examples. Finally, we present our concluding remarks in Section 5.

\section{Related Work}

The roots of physical hypermedia can be traced to the first mobile tourist guides, such as [2]. Even though not explicitly mentioned, this guide of the city of Lancaster mixed real world objects with their digital counterparts in the same was as PH software does. More recently, in [9] the authors present a powerful framework, HyCon, which supports not only context-aware physical navigation but also allows users to create their own linking structures and trails. In [11] meanwhile, an object-oriented framework (HyperReal) which allows mixing the real and the digital world is presented. Finally, in [10] the authors discuss the idea of "walking" a link and propose a future scenario in which the physical world will be augmented with digital information to make it more accessible to the traveler, particularly a disabled one.

Our research has been certainly inspired in these seminal research projects. There are some differences among our approach and the previously mentioned; first, following our previous work on model-based hypermedia software building [14], we consider the existence of an underlying hypermedia application, in the context of which we provide context-aware services. For example Figure 1 clearly shows a hypermedia schema, which besides has been built on top of a conceptual model (not shown in the Figure) using well-known viewing mechanisms. We also characterize real-world objects according to the role they can play to assist the user's journey; finally from an architectural point of view we chose not to build a software substrate from scratch but to extend a service-oriented architecture with hypermedia services; in this way we can integrate hypermedia behaviors with other context-aware services not directly related with navigation.

\section{Physical Hypermedia Services}

Physical Hypermedia applications are a particular kind of context-aware mobile software, which basically aims at enhancing real world objects with digital information and links; this kind of software provides one coarse grained service: HInformation which allows to access the physical object properties and links. Notice that this service is by definition location-aware because the information which it presents depends on the actual user's position. As indicated in Section 1, HInformation might also exhibit more elaborated context-aware features, by filtering the presented information according to the user's profile. The distinctive feature of PH applications however is the notion of physical navigation which underlies the whole user experience. While digital navigation has been widely explored in the literature on hypermedia and the Web (even for context-aware access [15]), research on service support for physical navigation is still in its infancy (See [10] for a good introduction to the problem).

Our research is focused on providing context-aware support for the navigation task. In the following sub-sections we introduce the core ideas of our approach. 


\subsection{A Model for the Hypermedia Traveler}

We have developed a conceptual framework (and implemented it as discussed in Section 4) for understanding context-aware services to aid physical navigation. The framework which has been inspired in [17] defines a set of roles which physical objects may play in the context of the user's journey. While the work in [17] aims to support handicapped users while navigating the Web, we tied to the original concepts in [12] to provide digital help for physical navigation.

The basic idea is simple: when we traverse a physical space (e.g. a city) we constantly face objects which are usually assigned pre-defined, standard roles (i.e. they exhibit certain behaviors). We know which "behaviors" we can wait from traffic lights, signals, street names, etc. However, if we have the chance to augment them with digital information to be presented to the mobile user, the possibilities are enormous and certainly appealing.

Our framework provides an open set of predefined roles which can be assigned to physical objects and a simple model of user navigation through the physical space. These roles exhibit a set of services (their behaviors) which basically allow them to provide different kind of information or guidance to the mobile user. Therefore, by making a physical object play one of these roles we can enrich its behavior according to the user's needs.

Table 1. Travel Objects Roles

\begin{tabular}{|l|l|}
\hline \multicolumn{1}{|c|}{ Role } & \multicolumn{1}{c|}{ Example } \\
\hline Navigation Point & Street, Corridor. \\
\hline Decision Point & Junction, Corridor, Intersection. \\
\hline Alert & Traffic Light, Signs \\
\hline Information Point & Information desk. Monuments \\
\hline Attention & Advertisements \\
\hline Landmarks & Distinguished Monuments \\
\hline Travel Memory & Route Plan \\
\hline
\end{tabular}

A list of the most important roles, according to the research in [17] is presented in Table 1. In [5], the authors use this classification of travel objects to find similar ones in the context of a Web page, in order to provide assistance to the blinded user. Instead, we aim to assign these roles to physical objects in such a way that the traveler (in this case the mobile user who is traversing a walking link) improves his tour.

Summarizing, when the user traverses the (physical) hyperspace by following links he faces a multitude of objects, which might play those roles shown in Table 1. As part of the role's behavior the objects will provide certain services to help the user complete his task (e.g. know a city, shop a product, etc). Additionally all objects will also provide a set of general purpose hypermedia services such as: CancelNavigation, MapUpdate, etc, which are fundamental for the trip.

The application designer might decide which roles a particular application object (or class) may play; he may refine the framework by adding new services to a role or 
eventually new roles according to the application domain. We next describe the rationale for assigning a role to a physical object in the context of physical hypermedia.

\subsection{Bridging Role Services with the User's Context}

A naïve analysis might conclude that certain physical objects only play some predetermined roles: for example we might never expect that a traffic light acts as an Information Point; however a technician, who repairs traffic lights, will presumably receive information about the object's functioning or the foreseen schedule of reparation. In fact, even the most simple real world object might play different roles (and therefore exhibit different services) according at least to:

- The application context in which it is being accessed (clearly, the technician is not using a tourism application).

- The current user's activity; e.g. whether he is traversing a link to reach a particular place, or if he is just wondering around without a plan in mind.

For the sake of conciseness and precision, we will focus on those services which depend on the actual user's activity.

In our approach, roles are assigned to physical objects dynamically according to the current user's state regarding navigation, which means that for two different users, the object might play (at the same time) different roles.

Suppose, as shown in Figure 2 a tourist in La Plata (user A) who, while being in front of the Museum, chooses a link that directs him to the Government House; while he is traversing the physical space to reach that place, he may interact with intermediate objects to receive assistance in his task. When he faces the Cathedral, and knowing that his actual task is to reach another place, the Cathedral plays the role of Decision Point which indicates him (as one of his services) that he must turn left to reach the target. Meanwhile, another tourist who is beginning his tour (User B in Figure 2), may be facing the Cathedral as regular node.

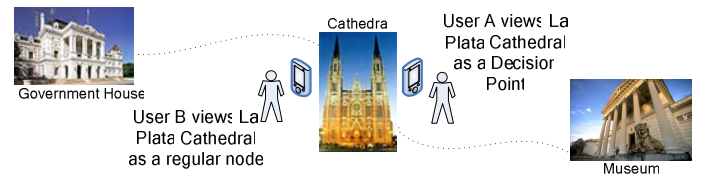

Fig. 2. Physical Objects play different roles according to the user's context

Summarizing, the set of services which a physical object provides depends on the role it is playing, which in turns depends on the current user's context. Additionally, other contextual information might be useful to further filter or elaborate the response of those services. For example, when viewing the Cathedral as a hypermedia object at visit hours, the tourist might be able to buy entrance tickets from his device, etc.

Next we describe how we extended an architecture for context-aware services to support the recording and use of the user's navigation state. The intent in Section 4 is twofold: we aim to show our solution and to emphasize how modularity allows seamless architectural evolution. 


\section{Architectural Support for Context-Aware Hypermedia Services}

In [13] we presented a pure object-oriented approach for specifying and providing context-aware services. The main focus of our presentation was to show how certain well-known design practices such as separation of concerns and treatment of context more as behavioral than as data constructs, helps to achieve seamless evolution when the original requirements change. In the context of our current research to enrich physical objects with context-aware role playing behaviors (in a hypermedia setting) the use of our "old" framework proved to be the best option as explained in the subsections below.

\subsection{The Architecture in a Nutshell}

A high level schema of the architecture presented in [13] is shown in Figure 3, together with the key hot-spots for adding physical hypermedia behaviors. We ignore the Sensing layer for the sake of conciseness.

In the Application layer, we specify application (digital) objects and behaviors in such a way that they are oblivious with respect to their physical nature or their eventual contextual features. This decoupling allows us to eventually extend legacy software (e.g. objects, behaviors and relationships in the application layer). In our specific domain, the application layer contains the classes which comprise the conceptual model in a hypermedia application [14]. Notice that relationships are considered firstclass citizen as they are the conceptual origin of hypermedia links. Hypermedia nodes, meanwhile are, as in OOHDM, views on conceptual classes.

Some objects of these classes will have a physical presence, and we need to sense when the user is in front of them. The Context Layer contains itself a set of sublayers, particularly a Location Layer which aim to extend conceptual classes with their location information. Location classes act as decorators on their conceptual counterparts and allow freeing the latter from the burden posed by location models (geometric, semantic, etc). This approach also allows adding physical presence at the instance, instead of class level; for example, some instances of class Monument might not have a physical presence, either because we decided not to provide information on them or because they no longer exist, though we still have digital information about them. This layer also contains "purely' physical objects which are relevant for the application though they have not been described in the application model, such as streets, traffic lights, etc. Additionally the context layer partitions the user's context in objects which represent each contextual element which is sensitive to the user dynamics. Physical hypermedia applications need to take into account the user's navigation context (as described in Section 4.2).

The Service layer contains the (context-aware) services that the system provides. Services, are specified as objects and attached to physical areas. The architecture uses dynamic publish/subscribe mechanisms to activate services. In the case of location-aware service as those described here, when the user changes his position, the object representing his position is updated triggering a message to the Service Environment, which in turn determines in which Service Area the user is located and thus which are the current available services. The main extension to the architecture is the specialization of the ServiceProvider component in order to change the way in which the available 


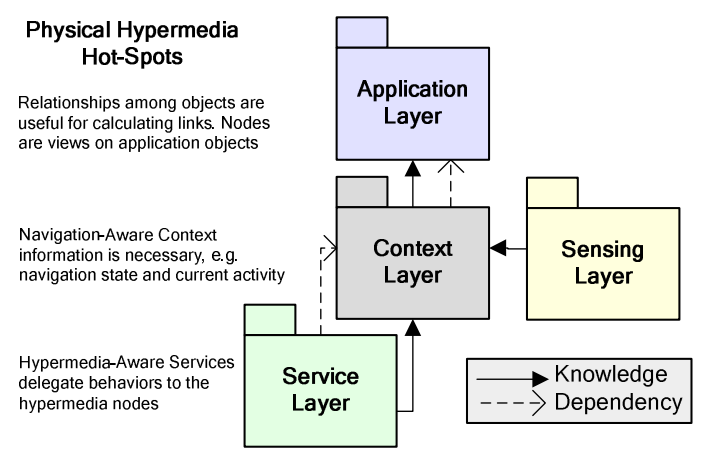

Fig. 3. High-Level View of our architecture

services are determined. This sub-class, named HypermediaServiceProvider, delegates to the physical objects (the "nodes" of the hypermedia network) the responsibility of returning the services which are meaningful in the actual context. This extension involves the assignment of roles to these objects as discussed below.

\subsection{Adding Hypermedia Contexts to Our Architecture}

The main two differences between the general idea of context-aware services and physical hypermedia services is that while the former are attached to places (locations) and further filtered according to the user's context, hypermedia services are allocated to the different roles played by physical objects; these role depends on the actual user's navigation context.

The simplest hypermedia service is triggered when the user selects a WLink; the response to this user's request, which is delegated to the source of the link (the physical object) may be a plan or map provided by the designer in which the source and target are highlighted; however this behavior can be overridden to provide more elaborated responses, e.g. according to the user's profile or preferences (e.g. if he likes walking), time of the day, etc.

The most interesting services however arise while the user traverses the physical space. Each time he faces a physical object, this object provides those services which are meaningful for the user; this is achieved by making the physical object play the role which corresponds better to the user's context. Figure 4 shows the Role hierarchy together with the relationship with the Physical Object base class.

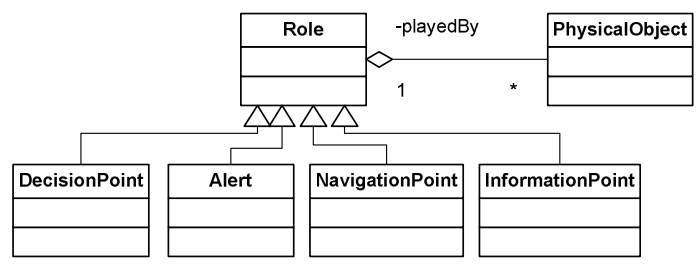

Fig. 4. Enhancing Physical Objects with Roles 
The assignment of roles to physical objects is a responsibility of a RoleBuilder object. The RoleBuilder analyzes the user context to determine these roles; in particular it analyzes the current user's activity and navigation state. In Figure 5 we show a simplified diagram of the possible user's activities and states. The user navigation state is represented as an instance of the State pattern [4] as partially shown in Figure 5 ; the navigation history is recorded as a list of traversed objects and navigation states. In a specific application, the designer may extend the State hierarchy if needed.

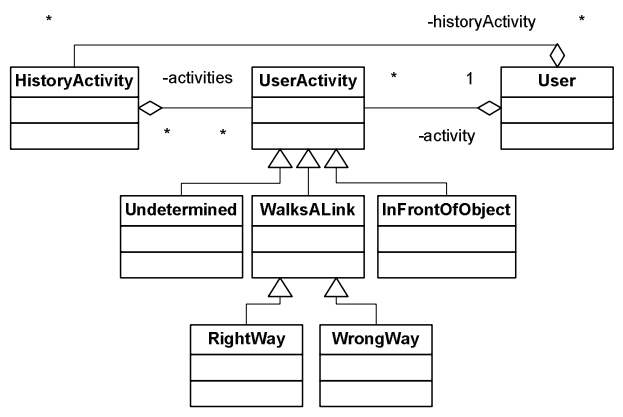

Fig. 5. User navigation state and history

RoleBuilder performs a "double dispatching" interaction with the actual user's activity object which may know which role corresponds to it. For example, if the user is walking a link and his actual position matches a "right way", the actual instance of RightWay in Figure 5 returns the Navigation Point Role object which is further assigned to the physical object. As a simplified example we show in Figure 6, the different kind of background information and services which a user who is moving through the city might receive when standing in front of the Cathedral. On the left, user A who is walking a link and is in the right way to his target; in the middle, user B who has lost his way to the target and on the right, user $\mathrm{C}$ who is standing in front of

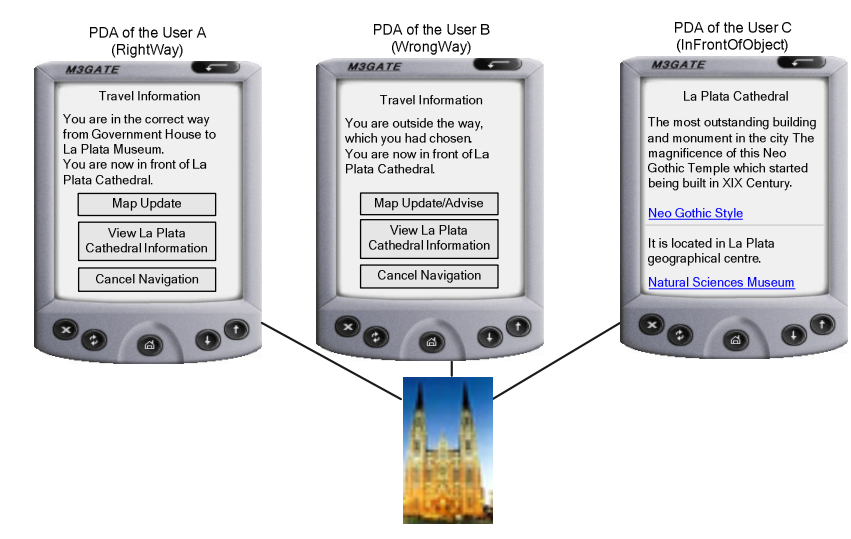

Fig. 6. Relationships among the user state and the response of physical objects 
the Cathedral but he is not following a link. In the first and second case the physical object assumes the role of a navigation object, with slight differences according to the actual user's state. In the last case, the physical object behaves as a regular node.

\section{Concluding Remarks and Further Work}

In this paper we introduced the concept of physical hypermedia services and showed why they must be provided according to the user's context. We discussed which kind of contextual information is relevant in these applications; particularly we analyzed why the user's state according to the navigation task is relevant to determine the kind of services he needs.

By adapting a taxonomy of travel objects to our domain, we defined a conceptual framework in which physical objects play roles according to the type of travel object they exhibit to the traveler. We have shown an implementation of these ideas by describing a seamless extension of an architecture for context-aware services to which we added the needed machinery to deal with physical hypermedia objects and to take into account the user's navigation state to assign roles to these objects. We are currently working on several research areas. First, we are extending the travel objects framework to comprise a broader set of objects which can be specialized according to different application domains; for example we are interested in analyzing the situation in which physical objects move as in [8]. In this sense it is interesting to see how navigation states evolve. We are also adapting the OOHDM approach by defining a set of reusable navigation classes (nodes and links) targeted to physical hypermedia. From the point of view of support tools we are adapting a well-known browser (Mozilla Firefox) to support physical, context-aware navigation in its main menus.

\section{References}

1. Challiol, C., Rossi, G., Gordillo, S., De Cristófolo, V.: Designing and Implementing Physical Hypermedia Applications. In Proceedings of the 2006 Workshop on Ubiquitous Web and System Intelligence (UWSI 2006), Springer Verlag, LNCS, forthcoming.

2. Cheverst, K., Davies, N., Mitchell, K., Friday, A., Efstratiou, C.: Developing a ContextAware Electronic Tourist Guide: Some Issues and Experiences. In Proc. of CHI 2000.

3. Espinoza, F., Persson, P., Sandin, A., Nystrom, H., Cacciatore, E., Bylund, M.: GeoNotes: Social and Navigational Aspects of Location-Based Information Systems. Proceedings of Third International Conference on Ubiquitous Computing (Ubicomp 2001), pp 2-17. Springer Verlag.

4. Gamma, E., Helm, R., Johnson, J., Vlissides, J.: Design Patterns. Elements of reusable object-oriented software, Addison Wesley 1995.

5. Goble, C. A., Harper, S., Stevens, R.: The travails of visually impaired web travellers. In UK Conference on Hypertext, pages 1-10, 2000.

6. Gordillo, S.E., Rossi, G., Lyardet, F.: Modeling Physical Hypermedia Applications. SAINT Workshops 2005: 410-413.

7. Gordillo, S., Rossi, G., Schwabe, D.: Separation of Structural Concerns in Physical Hypermedia Models. CAiSE 2005: 446-459. 
8. Gronbaek, K., Kristensen, J., Eriksen, M.: Physical Hypermedia: Organizing Collections of Mixed Physical and Digital Material. Proceedings of the 14th. ACM International Conference of Hypertext and Hypermedia (Hypertext 2003), ACM Press, 10-19.

9. Hansen, F., Bouvin, N., Christensen, B., Gronbaek, K., Pedersen, T., Gagach, J.: Integrating the Web and the World: Contextual Trails on the Move. Proceedings of the 15th. ACM International Conference of Hypertext and Hypermedia (Hypertext 2004), ACM Press. 2004.

10. Harper, S., Goble, C., Pettitt, S.: proXimity: Walking the Link. In Journal of Digital Information, Volume 5, Issue 1, Article No 236, 2004-04-07. http//jodi.ecs.soton.ac.uk/ Articles/v05/i01/Harper/.

11. Romero, L., Correia, N.: HyperReal: A Hypermedia model for Mixed Reality. Proceedings of the 14th ACM International Conference of Hypertext and Hypermedia (Hypertext 2003), ACM Press, 2-9.

12. Passini, R.: Wayfinding in Architecture. Van Nostrand Reinhold, New York, 1984.

13. Rossi, G., Gordillo, S.G., Fortier, A.: Seamless Engineering of Location-Aware Services. OTM Workshops 2005: 176-185.

14. Schwabe, D., Rossi, G.: An Object Oriented Approach to Web-Based Applications Design. Theory and Practice of Object Systems (TAPOS), Special Issue on the Internet, v.4\#4, October, 1998, 207-225.

15. UWA Project. www.uwaproject.org

16. Weal, M., Michaelides, D., Thompson, M., DeRoure, D.: The Ambient Wood journals: Replaying the experience. In Proceedings of Hypertext and Hypermedia. ACM Press, NY, 2003, 20--27.

17. Yesilada, Y., Stevens, R., Goble, C.: A foundation for tool based mobility support for visually impaired web users. In Proceedings of the Twelfth International Conference on World Wide Web, pages 422-430, 2003. 